thighs and without levering our tuberosities off the seat and on to the peak, and, further, it should provide means of escape for the soft perineal structures in case a jolt or voluntary alteration of position should tend to put pressure upon them. We want to avoid perineal pressure and anything which tends to riding forward off the broad part or to galling the buttocks or thighs. To meet these requirements I think it will be apparent to you that the surface of the saddle should be a plane and the saddle must be capable of a slight tilt backwards if the rider require such to keep him seated, and, secondly, a groove or opening for the perineum is adrisable, though not essential, for in the Brooks' B 90 saddle you will find as comfortable a saddle as you can wish for. Then saddles may be divided into suspension and treed saddles, and for ease and comfort the suspension have my preference. The Sans Bec is misnamed, as it has a beak.

Fig. 4.

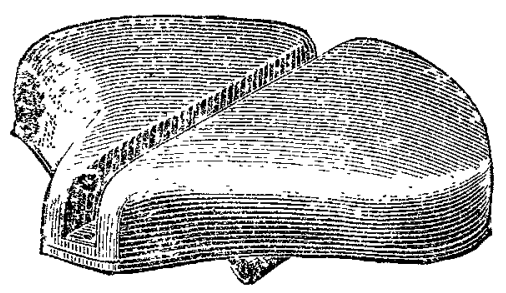

Sans Bec.

The peak is, it is true, reduced to a minimum and the sweep for the thighs is good. You will see that it is made on a tree which is perforated for the reception of symmetrical and ingeniously contrived rubber pads, which are placed on either side of a deep but too narrow central furrow. I expected to be comfortable on this saddle, but was not, and I think that the reason was that it was not roomy enough. I should get sore on it. It is a saddle which may be recommended to young men who care more for lightness and speed in riding than for actual comfort, and they need have no apprehension of perineal pressure. Suspension saddles are the various makes which are usually supplied and are usually faulty. There is, however, one which I mentioned just now, the Brooks' B 90,

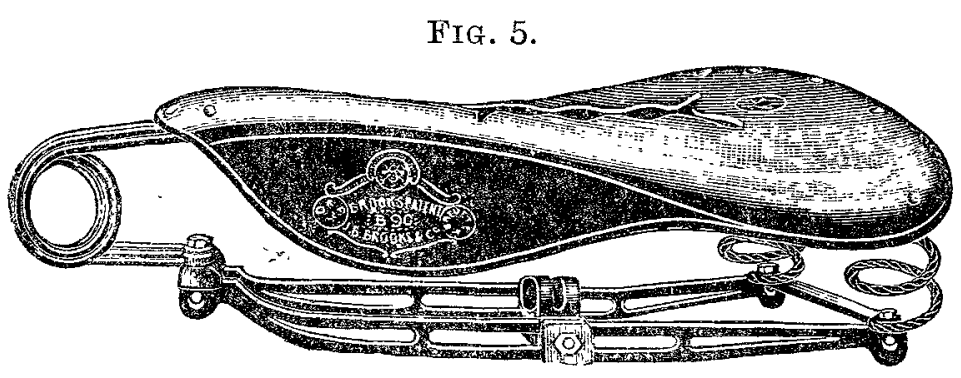

Brooks' B 90.

which is comfortable, and from its length practically safe. Furnished with an elastic, easy, spring, it is most comfortable to ride upon though it will never come into general use, I fear, on account of its size and weight; but to those riders who do not heed these matters it will be found a desirable seat and may be slightly tilted backwards with advantage.

I have worked at this saddle question for some time and have had various wooden ones carved, each with an opening for the perineum, and I have found them not uncomfortable to ride upon. I have, however, a liking for the suspension saddle and have had these two made by a smith and

FIG. 6.

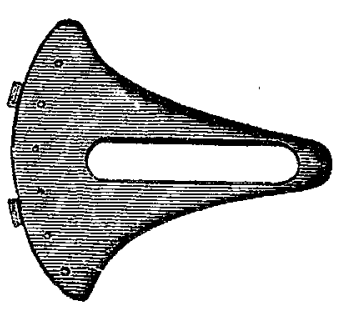

harness maker for $m y$ own use. You see that they are long and have an almost absolutely flat surface save only for the slight downward curve of the cantle. The peak has hardly any superior surface, being occupied almost entirely by an opening which runs back from the pommel and is preserved in one case by a metal fork and in the other by a wire. The peak, too, rotates round its horizontal axis and so provides a slight and comfortable amount of play with the thigh action.

This paper will be incomplete to a far more serious extent than it is if I omit all mention of women's saddles. I have had no case of injury to treat, but have been asked about chafing and soreness. As these occurred, without exception, in beginners one must not regard them too seriously. I think, however, that women are less liable than men to damage from pressure. They have not the exposed urethra, and have no prostate to be bumped about. Still they, or those who ride in skirts, have to use a short-peaked saddle, and I think that they would be as much benefited by a groove as men are. They do not ride as fast or as carelessly as men do, and so can somehow do better with a seat than we do. A word of practical advice to any who know of women who are beginning and have a fancy for the seat is that they ride on it as soon as they have learnt to keep on their machine, as the more they get accustomed to the saddle the more difficult they will find it to make the change. In conclusion, let me say that I can recommend no saddle as being universally applicable. I might as well recommend one pair of boots to fit everybody. Each rider has his peculiar method of sitting and pedalling, and each must select for himself a saddle which suits him. I do advise riders to try their saddles before they buy them, and to try them on a ride and not as they do a machine by a few turns up and down the street outside the agent's shop. They should understand thoroughly the right position of the saddle, which is such that they can, at the fullest extension required of the leg, sit properly upon the broad part of the saddle; and to obtain this the saddle must be low enough and far enough back, and probably in riding this position will be assisted by a slight raising of the peak. If the saddle is too high they are obliged to hang over the peak to reach the pedals, and if it is too far forward the leverage of the thighs throws them on to the peak and pommel. A good rule as to the height of the saddle is to see that at the point of greatest extension of the leg the heel can be brought to, or slightly below, the level of the pedal. Given a good saddle properly placed I am confident that only a few elderly riders need fear perineal pressure in cycling.

Exeter.

\section{HÆMORRHAGE DURING THE EARLY MONTHS OF PREGNANCY.}

BY AMAND ROUTH, M.D., B.S., M.R.C.P.LOND., OBSTETRIC PHYSICIAN WITH CARE OF OUT-PATIFENTS AT CHARING-CROSS HOSPITAL, PHYSICIAN TO THE SAMARITAN FREE HOSPITAL FOR WOMEN, ETC.

WHEN I was asked to read a paper at the South-West London Medical Society I thought over the various common obstetric and gynæcological ailments which most concern us as practitioners. In the special departments of medicine, obstetrics and gynæcology, the two symptoms which at once attract a woman's attention are pain and hemorrhage. As an isolated symptom in gynæcological cases, pain is more likely to lead a patient to her physician than hæmorrhage. It is this fact which acts so disastrously during the course of uterine cancer, most patients neglecting the early hæmorrhage and discharge, only seeking advice when pain is superadded. In obstetric cases, on the other hand, where a woman believes herself to be pregnant, the onset of hæmorrhage usually induces her to send for professional assistance. I therefore chose Hæmorrhage during the Early Months of Pregnancy as the subject for my paper, and did so with the hope of learning a good deal more from my audience than I can hope to personally impart. I do not propose to discuss the subjects of accidental hæmorrhage or that arising from placenta prævia, for these varieties are best recognised in the later months and are for all practical purposes indistinguishable from other cases of threatened abortion, even if they should cause hæmorrhage in the early months. Cases of hæmorrhage during the early months seem to naturally fall into two groups: (1) where the hæmorrhage is

I A paper read before the South-West London Medical Society on May 13 th, 1896. 
directly due to the existence of the pregnancy, and (2) where it is due to some condition independent of, but concurrent with, the existing pregnancy. The following may be taken as a rough summary of cases coming under the two classes: (1) threatened abortion, missed abortion, incomplete abortion, moles (apoplectic ovum, carneons mole, hydatidiform degeneration of chorion), decidual endometritis (diffuse, polypoidal, and catarrhal), and extra-uterine gestation; and (2) persisting menstruation, granular erosion of the cervix, adenoma of the cervix, mucous polypus of the cervix, fibroids, malignant disease, and ulcerations (specific and non-specific). As a rule, we should be on our guard against the patient being pregnant in all cases of hamorrhage between puberty and the menopause. Undoubtedly many mistakes are made from forgetting that a pregnant woman need not necessarily have amenorrhcea. It is very possible for a woman to be pregnant several months and yet to be either regular, or approximately so, or else to have metrorrhagia. So much is this the case that it would be safer to go to the opposite extreme and if a married woman complains of metrorrhagia-irregular hæmorrhages every day or every few days-assume for the time being that she is pregnant until this can be excluded. This mental reservation is often advisable in cases of unmarried women before the menopause, for it must be remembered that they may have special reasons for deceiving us as regards their menstrual history. I will now consider shortly the varieties named above in the first group.

Threatened abortion.- Here hremorrhage is usually the first symptom, but if the abortion is becoming inevitable pain soon supervenes, the cervix dilates, the ovim begins to protrude, and perhaps the membranes rupture. If protrusion has commenced partial separation must have taken place, and the abortion should be encouraged. Short of actual protrusion, where hæmorrhage is not severe, attempts may be made to prevent the abortion. I need not here speak of this. If the abortion be deemed inevitable a warm antiseptic douche should be given, and if hæmorrhage be slight, and especially if the pregnancy be under twelve weeks in duration, the case should be left to nature. If hæmorrhage be profuse plug the cervix and vagina with gauze and give ergot, or else, as I personally much prefer, put the patient under anæsthesia, dilate up the cervix by the rapid method if the cervix will not yield to the finger, and gently strip off the entire orum from the uterine wall, if possible without rupturing the sac, using the finger as a sensitive curette, meanwhile keeping the uterus firmly pressed down on to the inside fingers by means of the other hand. After removal of the ovum, douche out the uterine cavity with iodised water, and if hremorrhage be profuse plug the uterus with antiseptic gauze - a means which I have never known to fail.

Missed abortion. - These cases are not always easy to recognise. A woman misses a period or two, followed maybe by a rather sharp loss, and may then begin to lose a little every day or once a week, or even have gushes of blood approximately every month, and, excusably enough, has no idea that her uterus contains an ovum. In some cases the ovum thus retained is changed, by extravasation of blood into its tissues, so that it becomes an apoplectic ovum, and as such will be considered later. The diagnosis of a retained dead ovum is made chiefly by the fact that one or two periods have been missed, by the presence of irregular hæmorrhages, and by the fact that the uterus is smaller than it should be, dating from the last regular period, and that, moreover, it is not enlarging, but tending rather to decrease in size. It has to be distinguished from subinvolution of the uterus after an early abortion, from incomplete abortion, or from molar pregnancy, and it may at first be impossible to be sure; but fortunately in all these cases ergot may be given, and its influence upon the uterus under these several conditions will go far to enable a diagnosis to be made. If, however, hæmorrhage persist, or the temperature rise, rapid dilatation should be effected under anæsthesia, the cavity explored, a complete diagnosis thus made, and the condition found at once dealt with. Cases of missed abortion, apart from those which result in the formation of moles, most usually occur when the ovum dies, as it were, very slowly, a lingering death from some lack of vitality either in the impregnating spermatozoon, as in cases of syphilis, Bright's disease, diabetes, or lead poisoning in the husband, or from similar or other chronic blood states such as anæmia in the woman. In such cases the uterus becomes tolerant to the presence of the degenerating and eventually dead ovum and may not at once throw it off. In such cases, also, the uterus would be less vascular and consequently less active. On the other hand, when the ovum dies more suddenly, an immediate abortion follows, except where extravasation of blood or decidual endometritis has made the membranes unduly adherent. Sudden death of the ovum is due to high temperature or to blood poisoning, as in acute specific fevers or in cholera \&c., or to imperfect aeration of blood in cases of pneumonia \&c. The following case shows how serious even a short retention may become.

CASE 1.-A married woman, seen with Mr. Busfield of Clapham, had had two children, the last eight years previously. She had been quite regular, her last period having been fourteen days ago. On April 3rd she began to lose a little blood, and this continued slightly for three days, and during the same period her temperature rose and rigors occurred. Though the history was very misleading Mr. Busfield suspected a missed abortion, and on the fifth day I saw the patient with him. She had then just had a rigor and her temperature was $106^{\circ} \mathrm{F}$. Occasional uterine colic was present, but no other discomfort. Under anæsthesia an intact six weeks' ovum was removed, and in one and a half hours her temperature was $103^{\circ}$, in three hours $99.5^{\circ}$, and it was normal next morning. Hæmorrhage was rather free after removal of the ovum, requiring plugging of the uterine cavity with gauze.

The following case had less severe symptoms, but was remarkable from the ovum having died within a few days of reaching the uterine cavity.

CASE 2.-A married woman aged twenty-eight years, who had bad one child seven years previously, aborted in March, 1895. She was quite regular till her September period, 1895, when she lost rather profusely, and continued to lose almost daily for the following five weeks, when, finding that the uterus was only slightly enlarged, to be not increasing in size, and that the hæmorrhage was getting worse, I rapidly dilated and found an ovum of about ten days loosely attached to the left cornu. She lost rather freely for ten days, but has since been quite regular. This very early ovum had apparently been conceived about ten days before the September period, had not been thrown off when that period occurred, and had been retained for another six weeks.

CASE 3.-A foetus [which Dr. Routh showed] had been retained in utero for five months, causing metrorrhagia and septicamia. Finally the uterus was rapidly dilated by Dr. Gibson Berkeley of Camberwell, the foetus removed by the finger and the sloughing membranes by the curette, the woman doing well. The fotus had undergone mummification and partial calcification.

Incomplete abortion.-If hæmorrhage or sepsis occur after a presumed abortion and the uterus is not increasing in size the cervix should be rapidly dilated and its cavity explored. True septicæmia in such cases is rare, sapræmia or septic intoxication being the usual cause of the pyrexia, and it is as a rule found that removal of the decomposing product causes the temperature at once to drop and the hæmorrhage to cease. The technique of the operation is as follows. The essentials are (a) rigid antisepsis, (b) dilatation so as to admit the finger, $(c)$ complete evacuation of the uterine contents, and (d) anæsthesia is almost an essential. After washing the vulva and vagina with wool soaked in corrosive sublimate solution, seize the anterior lip of the cervix with a vulsellum forceps with blunt teeth like Teale's forceps. If the finger cannot be inserted dilate rapidly with graduated bougies and explore the whole cavity very carefully, especially the cornua, pressing the uterus down from outside. Remove any pieces found adberent with the finger if possible, or with the forceps or blunt curette as may be most convenient, but be careful not to injure the uterus, the tissues of which are very soft after abortion. For hæmorrhage apart from sepsis the curette is hardly ever required and in very rare cases should the curette be a sharp-edged one. If the piece removed be offensive the uterus should be irrigated with 1 in 2000 corrosive sublimate solution. If, as often happens when a piece of placenta is removed, very free hæmorrhage occur, the uterus should be at once plugged with iodoform gauze, a process which in my experience has in these cases never failed to arrest the bleeding. The gauze should be removed in twenty-four hours. Most cases of incomplete abortion are easy to diagnose, owing to the history of amenorrhœa, followed by hæmorrhage, passage of the foetus, and subsequently more or less continuous losses. Where these points are denierl the diagnosis is impossible without an exploratory dilatation, as in the following case. 
CASE 4. - A woman who had been married three years had borne one child two years ago and had never aborted. Her present illness began with rather profuse hæmorrhage six months before, commencing fourteen days after a natural period. Since then she had lost blood daily, with no increase by which a menstrual date could be fixed, though she had had several severe floodings at irregular intervals. She came under the care of Dr. Murphy of Twickenham six months after the first hæmorrhage, and with his assistance I explored the uterus and removed a piece of placenta which had undergone some fibroid change, and had presumably been there for at least six months, though her view of it was that it had been there since her previous labour two years before. If so, it had produced no symptoms for the first eighteen months-a very unlikely alternative.

The following case is one of a rare condition where the placenta and membranes had come away and the foetus had been retained. It was most probably a case of placenta prævia where the placenta from some accidental cause had become prematurely detached.

CASE 5.-A married woman aged twenty $\cdot$ six years had had three children, the last eleven months previously; two abortions had occurred prior to the birth of the last child. Amenorrhcea occurred from March 1st to May 3rd. She then "flooded" and had severe pain and passed several "pieces of tesh." Since then (six weeks) she had lost blood daily with occasional fotor. She suffered much from backache, general malaise, and nausea. On examination the uterus was found to be bulky, flabby, and four and a half inches long, and I believed it was a case of abortion with probably a retained piece of placenta and put her on ergot till arrangements could be made for dilatation. In two or three days she had a series of sharp pains and passed a somewhat shrivelled, macerated fotus of about three months' gestation with no clots, and digital exploration of the uterus proved that the afterbirth had also come away, probably over three months previously.

Molar pregnancy.-The " moles" most ordinarily met with are "blood moles" or " apoplectic ova," which are due to hæmorrhages into the decidua or between the decidual layers. Less frequently "fleshy moles" are found, due generally to hæmorrhages which have become organised or which are complicated with a good deal of diffuse or polypoidal decidual endometritis. These moles may remain in atero for many months, usually acting as foreign bodies and keeping up daily or irregular hæmorrhages. Sometimes the aterus becomes tolerant to their presence and menstruation may for a few periods become regular, but sooner or later the mole will be expelled with smart hrmorrhage and some pain, which latter symptom may, however, be almost entirely absent. Apoplectic ova are probably instances of the commonest pathological changes of the early ovum.

CASE 6.-[Dr. Routh showed a specimen of an apoplectic ovum which was expelled seven months after conception.] The woman had not been "unwell" for five weeks, when some hæmorrhage occurred, and doubtless at the same time hæmorrhage also occurred into the envelopes of the ovum itself, leading to the death of the foetus. At first I diagnosed missed abortion and put her on ergot. All irregular losses then ceased and she menstruated regularly and I thought I had been mistaken, but at the seventh month the orum was expelled with some hæmorrhage and practically no pain.

CASH 7.--In June, 1891, I saw a patient with Dr. Dodson of Wandsworth with a history of having had no regular period for six months, but she had had for over four months a daily grumous discharge, which for the last four weeks had gradually given place to offensive watery discharge. On examination the uterus was found equal to the size of a three months' pregnancy, and we came to the conclusion that the foetus had died in the second or third month and was now retained, and had undergone some organic change and was probably a fleshy mole. As, however, the diagnosis was uncertain we gave her ergot on trial, and the hæmorrhages ceased, but in September-i.e., ten months after conception-the mole came away spontaneously.

Hydatidiform degeneration of the chorion-" "hydatid mole."-This can only be diagnosed with certainty by the discovery of cystic villi in the discharges, which are mainly watery blood, but it may be strongly suspected if the uterus is a good deal larger than it should be at the date to which we believe the pregnancy is advanced. This is just the opposite to what occurs with apoplectic ova or fleshy moles, where, though we gather that the woman should be three or four months pregnant, we find the uterus about the size of six or eight weeks. These hydatid moles may also remain in utero many months. If hæmorrhage be severe the uterus should be emptied, trying to do this with ergot first, and, failing that, by rapid dilatation. If hæmorrhage is slight, and yet the diagnosis is sure owing to vesicles having been detected, we should not be too much in haste to empty the uterus, as twin pregnancy may be present, only one of which is bydatid.

CASE 8. - [Dr. Routh showed a specimen of hydatid mole from a patient who was sent to him by Mr. Stanley Boyd.] She had had constant daily hæmorrhage for two months after two and a balf months' amenorrhcea. The uterus was the size of a six months' pregnancy. Rest in bed made no difference, and as the discharge became offensive and the temperature rose full doses of ergot were given and this mole came away, some fragments in addition having to be digitally removed subsequently. Two years later she had a bealthy child.

Decidual endometritis.-Next to apoplectic ova the commonest pathological change in the early ovum is decidual endometritis-diffuse, polypoidal, and catarrhal. Of these the catarrhal form is the most frequent, especially in women who have previously bad subinvolution and chronic endometritis. Not infrequently one is able to trace a syphilitic taint, which in a few cases has been congenital. In such cases women may lose a little blood-stained or brownish discharge and as a rule do not believe themselves to be pregnant. After a few weeks the discbarge may become watery and gushes of clear or straw coloured water come away, being one form of hydrorrhcea gravidarum. These cases give rise to more mistakes in diagnosis than either moles or the other varieties of abortion already considered, but if a careful bimanual examination be made the diagnosis is not difficult. The uterus is found to be about the size we should expect in a normal pregnancy of the same duration, not larger, as in hydatid mole, and not smaller, as in cases of missed abortion or fleshy mole. The examination is made the more difficult because, owing to the unhealthy state of the uterus, its upper limit cannot always be felt and its intermittent contractions are very transient. If ergot be given incautiously or the uterus be roughly handled the uterus, already holding its contents very loosely, expels the ovum. These cases have also to be distinguished from both subinvolution and fibroids. Most of these cases go on to full term if treated by three or four grains of iodide of potassium thrice daily. This drug seems to be almost a specific remedy, though it is impossible that all cases can be syphilitic. The most typical cases of decidual endometritis occur in small-pox, and, owing to the hæmorrhage, have been called "hæmorrhagic endometritis," but these are exceptional in usually ending in abortion. The following case occurred after the menopause had apparently set in, rendering the diagnosis somewhat obscure.

CASE 9.-A patient aged forty-five who had been married twenty years had had eleven children, the last three years previously. Menstruation was regular till March, 1891, then amenorrhœa supervened till August, 1892, when she "flooded," and had in January, 1893, for over five months lost blood almost daily. Pregnancy of five and a half months was diagnosed, and she was put on iodide of potassium to cure the endometritis. The hæmorrhage soon ceased, occasional gust es of clear water passing painlessly. She was confined on April 15th of a healthy child, but the placenta being pathologically adherent from the endometritis had to be stripped off.

Extra-uterine pregnancy (mainly tubal).-This cause of hæmorrhage must not be omitted, though relatively rare. One or two periods are missed, sometimes only one; then irregular hæmorrhages occur, and possibly a uterine decidua, differing from that of an intra-uterine pregnancy in having no chorionic villi, is passed. Physical examination reveals a sympathetically enlarged uterus and a tumour on one or other side or in Douglas's pouch. Possibly also the patient may complain of pain-the pain cf tension-on that side also. The treatment is to remove the tube as soon as the diagnosis is sure. If rupture has already occurred downwards into the broad ligament nothing operative need be done, as the hrmorrhage is necessarily bound down. If rupture has taken place into the peritoneal cavity, and it can be felt to be encysted by pre-existing bowel adhesions which roof over the pouch of Douglas, we may delay operation hoping for permanent arrest of bleeding; but if no localised hxmatocele be felt an internal hæmorrhage is evidently going 
on, the abdomen must be opened at once, and the ruptured tube removed.

The second large group of hamorrhages during the early months of pregnancy consist of those varieties due to conditions concurrent with and complicating the pregnancy.

Persisting menstruation is fairly common during one, two, or three months, and in the last case the uterus would be found to correspond in size to a gestation of three months longer duration than was indicated by the amenorrhoca. Physical examination would distinguish these cases from hydramnios, and the absence of irregular losses would prove them not to be hydatid moles. The most likely mistake to fall into is that the large uterine tumour may at the end of the three months' pregnancy be taken for a fibroid tumour. Time, however, will always clear up the case.

I will now very briefly consider some of the local complications which cause hemorrhage during early pregnancy. Granular erosion of the cervix. - Here the hamorrhage is slight and as a rule only present after coitus.

Adenonza of the cervix.-If a granular erosion has been present before conception there will have been a denudation of the squamous epithelium of the raginal portion. The coexisting glandular cervicitis is aggravated by the increased vascularity of preonancy, and the resulting projections of papillary processes look like the tentacles of a blood-red sea-anemone. Such a cerrix bleeds freely when touched, and when not touched there is usually a blood-stained leucorrhcea. This condition disappears spontancously after delivery, and can be held in check during pregnancy by usual local remedies.

CASE 10.-A married woman aged thirty years bad had one child four years ago. She aborted eighteen months arro. She had had severe endo-cervicitis and erosion. She hart now had amenorrhoea for two months, except that she always lost blood after coitus and had a copious leucorrhcea, occasionally blood-stained. The uterus was found to be retroverted and gravicl. The cervix was bright red, bled easily on contact, and was evidently adenomatous. The uterus was reduced and the cervix painted a few times with iodised phenol. She went to full term and did well.

Mucous polypus of the cervix.-A woman may perhaps be suffering from almost daily loss of blood, yet becomes pregnant and continues to lose blood as before. The uterus is found to be enlarged, and, naturally enough, if great care be not taken the diagnosis of rapidly growing fibroid is made, and the mucous polypus, even if noticed, is then looked upon as an unimportant complication.

CASE 11. - A woman aged forty-one years had been married four months. She had slight irregular losses before marriage and more severe and frequent since. She was thought to have a rapidly growing fibroid requiring operation. She was found to be about three and a half months pregnant and had two small mucous polypi hanging from the endocervix. These were burnt off and her child was born in due course.

Maliqnant disease of the cervix.-I do not know of any case of pregnancy occurring where pre-existing cancer or sarcoma of the body of the uterus was present, but it is possible that some of the cases of deciduoma malignum recently reported may be thus explained. ('ancer of the cervix and pregnancy are not nearly so rare, and it is evident that with such a cause of hæmorrhage existing, a mistake in diagnosis may be easily made.

CASE 12.-A woman came to the out-patient department of Charing-cross Hospital suffering from metrorrhagia of some months' duration. 'This was found to be due to epithelioma of the cervix, already beginning to slough and ulcerate. She had a large uterus, but the question of pregnancy did not occur to me. She was admitted and I removed the cervix as high as the internal os uteri. and even then had no suspicion that she was over two months' pregnant. She disappeared from under observation, but I subsequently heard that she went to full term, that the epithelioma speedily recurred, as it always tends to do during pregnancy, and that during a temporary closure of the hospital she went to St. Thomas's Hospital, and Dr. Cullingworth successfully performed Cxsarean section, the woman dying some months after from the malignant disease.

These few cases and my imperfect comments will tend, I hope, to show how important a careful and cautious diagnosis is in all instances of metrorrhagia where pregnancy is a possible explanation or concurrency.

Manchester-square, $W$.

\section{THE TOXINE THEORY OF SYPHILIS.}

By ARTHUR H. WARD, F.R.C S. ENG.

SURGKON 'TO OUT-PATIENTS, LONDON LOCK HOSPITAT; SURGICAT REGIS'LAR 'WO ST, GEOR(TIE'S HOSPITAL.

IN a paper on the Pathology of Syphilis ${ }^{1}$ I advanced a working hypothesis deduced from a consideration of the phenomena of the hereditary disease, and I have recently applied it to the question of latency in syphilis. ${ }^{2}$ In order to cover the vast array of facts involved it is evident that such a hypothesis must be of elemental simplicity. Reduced to its lowest terms it may be stated thus : (1) syphilis is associated with the growth of a microbe in the organism; and (2) this microbe produces a toxine which is the active cause of the phenomena of syphilis. In the light of modern bacteriology this theory seems almost self-evident; however, until the specific microbe has been discovered, cultivated, and inoculated, and the toxine isolated, it must remain a hypothesis only. I will endeavour to show that, at any rate, it is one that will work. The toxine must be supposed to share the properties of other microbial toxines; for instance, it is diffusible and follows the laws of osmosis, it is irritating to the tissues and causes local inflammatory reaction, it is strongly chemotaxic and attracts leucocytes into the area in which it is present, and when existing in quantity in the circulation it causes symptoms of general toxæmia.

The first thing to consider is the point of original infection, the abrasion or fissure through which inoculation takes place. This is often so minute as to escape notice, and when more extensive-for instance, a torn franum-the mere fact of its occurrence will put a stop to further exposure to the infection. It is, therefore, plain that only a few microbes can be implanted in the first instance, while the prolonged course of the disease indicates that the infection is a slowly developing one. Hence, although there is no reason why the primary colony should not start growing and forming toxine at once, yet it will be so small, or so sparsely sown with microbes, that the toxine produced will be inappreciable in amount Chemotaxis will occur from the first, but three weeks may well elapse before sufficient toxine has been developed to cause a cell infiltration dense enough to distend the intercellular spaces and be visible to the naked eye. Thus the primary incubation period is one of apparent latency. When the suaces in which the colony is growing have become sufficiently crowded up with leacocytes the indurated papule, the first sign of syphilis, will result. The induration is the essential feature of the lesion, since ulceration is not in variable; this, however, almost always occurs. I believe it to be due to the degeneration of the invading cells from overcrowding and consequent mal-nutrition for the following reasons. Microscopical examination shows that the walls of the vessels are thickened and their calibre reduced by the presence of round cells-cells, I believe, in the act of invading the colony in response to the stimulus of the toxine; there fore, the blood-supply of the infected area is defective Further, the surface of the early bard sore presents a whitish colour and is covered with a number of small, irregula depressions ; it looks worm-eaten-"vermoulu," as Ibsen has it ; these depressions can only be due to the disintegration of collections of cells massed among the fibres of the cutis Again, when the primary induration consists of several con fluent papules, ulceration occurs at several points correspond ing to the centres of the several colonies, where the indura tion would be densest and degeneration most likely to occur these ulcers ultimately fuse into one large sore. Lastly, when the primary sore is situated on a vascular part, like the lip, very little ulceration generally occurs, although induration may be most extensive. The worm-eaten appearance dis appears in a few days and the sore becomes smooth and callous, because, I think, it must form a nidus for sapro phytic microbes, whose toxic products will dissolve the superficial layers of tissue, as in other chronic ulcers. When the sore is beneath the prepuce, and the discharge retained evidence of the presence of such toxic irritants occurs in the shape of acute irritation, suppuration, and possibly phagedæna. In the normal hard sore no suppuration exists because it is formed by disintegration only, as I have tried to show.

Since the primary colony is single, and there is no other 